\title{
Safety of biologics in inflammatory bowel disease patients with COVID-19
}

\author{
Simcha Weissman ${ }^{1}$ (D) Muhammad Aziz ${ }^{2} \cdot$ Wade-Lee Smith $^{2} \cdot$ Sameh Elias $^{1} \cdot$ Arun Swaminath $^{3}$. \\ Joseph D. Feuerstein ${ }^{4}$
}

Accepted: 8 June 2021 / Published online: 15 June 2021

(c) The Author(s), under exclusive licence to Springer-Verlag GmbH Germany, part of Springer Nature 2021

\begin{abstract}
Background Patients with Inflammatory bowel disease (IBD) remain highly concerned that either their disease or medicationsnamely, biologics - may increase the risk of severe coronavirus-2019 (COVID-19). We aimed to assess the safety of biologics in Inflammatory bowel disease (IBD) patients with COVID-19.

Methods We systematically reviewed multiple databases to find relevant articles reporting the effect of biologics on "severe" COVID-19 in IBD patients. Those in the form of case series (>10 patients), case-control, and cohort studies were included. Severe COVID-19 was defined as intensive care unit (ICU) admission, mechanical ventilation, and/or mortality. Pooled analysis with multivariate regression was performed.

Results A total of 12 studies with 2681 patients were included. The proportion of females was $(48.3 \%, 95 \%$ confidence interval (CI) 47.0-49.5\%). The proportion of UC patients was (44.8\%, 95\% CI 41.0-48.5\%). Overall, in IBD patients, the need for mechanical ventilation, intensive care unit (ICU) admission, and mortality was $5.1 \%, 6.1 \%$, and $4.5 \%$, respectively. Use of biologics did not show a moderating effect on mechanical ventilation $(p=0.68)$, ICU admission $(p=0.27)$, or mortality $(\mathrm{p}=0.20)$.

Conclusions Our findings advocate for the continued biologic therapy in IBD patients during the COVID-19 pandemic. Nevertheless, the incidence, severity, and outcomes related to COVID-19 in IBD patients' needs to be reassessed as data continues to emerge.
\end{abstract}

Keywords Biologics $\cdot$ COVID-19 $\cdot$ SARS-CoV-2 $\cdot$ Inflammatory bowel disease $\cdot$ Mortality

\section{Introduction}

Novel coronavirus disease-2019 (COVID-19), caused by severe acute respiratory syndrome coronavirus-2, originated in Wuhan, China, and spread rapidly resulting in a global pandemic [1]. Both clinicians and inflammatory

Simcha Weissman

Simchaweissman@gmail.com

1 Department of Medicine, Hackensack Meridian Health Palisades Medical Center, North Bergen, NJ, USA

2 Division of Gastroenterology and Hepatology, University of Toledo Medical Center, Toledo, OH, USA

3 Division of Gastroenterology, Inflammatory Bowel Disease Program, Lenox Hill Hospital, Northwell Health, New York, NY, USA

4 Division of Gastroenterology, Beth Israel Deaconess Medical Center, Harvard Medical School, Boston, MA, USA bowel disease (IBD) patients remain highly concerned that either their disease or medications-namely, biologics-may increase the risk of severe adverse outcomes from COVID-19, given their increased propensity for infection [2]. We performed a systematic review and meta-analysis of the available literature to assess the safety of biologics in COVID-19 patients with IBD.

\section{Methods}

We performed a systematic review of the databases PubMed/ Medline, Embase, Cochrane, Web of Science, LitCOVIDNIH, and WHO COVID-19 from January 1 to November 3,2020 , to identify relevant articles reporting outcomes in IBD patients with COVID-19. Those in the form of case series ( $>10$ patients), case-control, and cohort studies were included. Studies were excluded if they did not report the 
A

Study name

Alloccaeta.

Attandi e d.

Axelrodeta.

Bezzio etal.

Berner et $d$.

Derikxt a

Luknet al.

Rodriguez-Lagoet d.

Taxonera et al.

Unga o etal.

$\underline{\text { Study name }}$

Allocca et al.

Attauabi et al.

Avelrod at al.

Eremer et al.

Derikxet al.

Guerraet d.

Lukinet $a$.

Rodriguez-lago et al.

Taxoner a et $\boldsymbol{d}$.

Unga o et $a$.

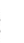

Study name

\section{Allocca etal.}

Attauabi a al.

Avelrod et al.

Bezzio et al.

Brerner et al.

Derikxet $\mathbf{a}$.

Gerra et al.

Hormati et al.

Lukin et al.

Rodríguez-Lago et al.

Taxcneraet al.

Ungaroet $\mathbf{a}$.
Statistics for each study

$$
\text { Event Lower Upper }
$$$$
\text { rate limit limit Z-Value } p \text {-Value }
$$

$\begin{array}{lllll}0.031 & 0.002 & 0.360 & -2390 & 0.017 \\ 0.039 & 0.013 & 0.115 & -5.418 & 0.000 \\ 0.012 & 0.002 & 0.081 & -4.380 & 0.000 \\ 0.025 & 0.006 & 0.096 & -5.097 & 0.000 \\ 0.040 & 0.026 & 0.061 & -14.269 & 0.000 \\ 0.070 & 0.034 & 0.140 & -6.600 & 0.000 \\ 0.138 & 0.078 & 0.231 & -5.656 & 0.000 \\ 0.012 & 0.001 & 0.167 & -3.088 & 0.002 \\ 0.083 & 0.012 & 0.413 & -2296 & 0.022 \\ 0.046 & 0.036 & 0.058 & -24.086 & 0.000 \\ 0.051 & 0.035 & 0.074 & -14.384 & 0.000\end{array}$

Event rate and $95 \% \mathrm{Cl}$

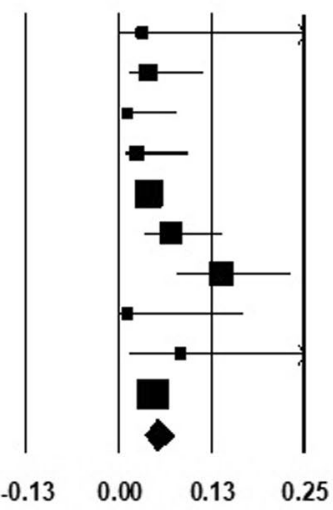

Event rate and $95 \% \mathrm{Cl}$

\begin{tabular}{ccccc}
$\begin{array}{c}\text { Event } \\
\text { rate }\end{array}$ & $\begin{array}{c}\text { Lower } \\
\text { limit }\end{array}$ & $\begin{array}{c}\text { Upper } \\
\text { limit }\end{array}$ & \multicolumn{3}{l}{ Z-Value } & p-Value \\
0.031 & 0.002 & 0.350 & -2390 & 0.017 \\
0.066 & 0.028 & 0.149 & -5.734 & 0.000 \\
0.012 & 0.002 & 0.081 & -4.380 & 0.000 \\
0.046 & 0.031 & 0.067 & -14.542 & 0.000 \\
0.100 & 0.056 & 0.176 & -6.592 & 0.000 \\
0.012 & 0.006 & 0.081 & -4.368 & 0.000 \\
0.138 & 0.078 & 0.231 & -5.656 & 0.000 \\
0.012 & 0.001 & 0.167 & -3.088 & 0.002 \\
0.083 & 0.012 & 0.413 & -2296 & 0.022 \\
0.057 & 0.046 & 0.070 & -24.678 & 0.000 \\
0.061 & 0.042 & 0.080 & -13.812 & 0.000
\end{tabular}

$$
\begin{array}{lllll}
-0.25 & -0.13 & 0.00 & 0.13 & 0.25
\end{array}
$$

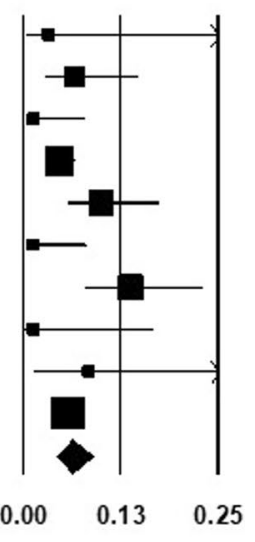

Event rate and $95 \% \mathrm{Cl}$

\section{Event Lower Upper}

rate limit limit Z-Value p-Value

$\begin{array}{lllll}0.031 & 0.002 & 0.350 & -2390 & 0.017 \\ 0.053 & 0.020 & 0.132 & -5.627 & 0.000 \\ 0.012 & 0.002 & 0.081 & -4.380 & 0.000 \\ 0.076 & 0.036 & 0.159 & -5.884 & 0.000 \\ 0.030 & 0.019 & 0.049 & -13.627 & 0.000 \\ 0.130 & 0.077 & 0.211 & -6393 & 0.000 \\ 0.012 & 0.002 & 0.081 & -4.368 & 0.000 \\ 0.003 & 0.000 & 0.051 & -4.029 & 0.000 \\ 0.025 & 0.006 & 0.094 & -5.116 & 0.000 \\ 0.050 & 0.013 & 0.179 & -4.059 & 0.000 \\ 0.167 & 0.042 & 0.477 & -2078 & 0.038 \\ 0.034 & 0.026 & 0.045 & -23.015 & 0.000 \\ 0.045 & 0.028 & 0.071 & -12.246 & 0.000\end{array}$


४Fig. 1 Forrest plot demonstrating the overall prevalence of outcomes-A need for mechanical ventilation, $\mathbf{B}$ need for intensive care unit (ICU) admission, and $\mathbf{C}$ overall mortality-in COVID-19 infected inflammatory bowel disease (IBD) patients

outcomes of interest (intensive care unit (ICU) admission, mechanical ventilation, and mortality) or excluded data on IBD medications (biologics). Pooled analysis was performed using a random-effects model. Study heterogeneity was assessed using the $I^{2}$ statistic. Multivariate regression model was applied using the proportion of patients on biologics, mean age, proportion of patients with ulcerative colitis (UC) and proportion of patients with Crohn's disease (CD) as moderates.

\section{Results}

The initial search yielded a total of 81 articles, and after screening for duplicates and excluding irrelevant articles, a total of 12 studies with 2681 patients were finally included. Two studies were prospective and 10 retrospective. Eight studies were single-nation studies (three from Spain, two from the USA, one from Italy, one from the Netherlands, and one from Iran) and four multinational. The study publication dates ranged from April 3 to October 20, 2020. The proportion of females was $(48.3 \%, 95 \%$ confidence interval (CI) $47.0-49.5 \%)$. The proportion of UC patients was (44.8\%, $95 \%$ CI $41.0-48.5 \%)$.

We found the overall prevalence of outcomes for all IBD patients as need for mechanical ventilation: $5.1 \%$ (95\% CI $3.5-7.4 \%, I^{2}=52.1 \%$ ) (as reported by 10 studies), need for ICU admission: $6.1 \%$, (95\% CI $4.2-8.8 \%, I^{2}=54.8 \%$ ) (as reported by 10 studies), and overall mortality: $4.5 \%$ (95\% CI $2.8-7.1 \%, I^{2}=68.0 \%$ ) (across all 12 studies) (Fig. 1).

Use of biologics (coefficient $-0.01,95 \%$ CI $-0.08-0.05$, $\mathrm{p}=0.68$ ) and mean age did not show a moderating effect on the need for mechanical ventilation (Fig. 2). The increasing proportion of UC (coefficient $-0.07,95 \%$ CI $0.15-0.00$, $\mathrm{p}=0.05$ ) and CD (coefficient $-0.06,95 \% \mathrm{CI}-0.09$ to -0.02 , $\mathrm{p}=0.002$ ) showed a significantly negative trend towards a need for mechanical ventilation. The R2 analog was 1 or $100 \%$.

Use of biologics (coefficient $0.03,95 \%$ CI $-0.02-0.08$, $\mathrm{p}=0.27$ ), mean age, and UC did not show moderating effect on ICU admission (Fig. 2). CD proportion showed a negative trend towards ICU admission; however, this was not significant (coefficient $-0.04,95 \% \mathrm{CI}-0.09-0.00, \mathrm{p}=0.07$ ). The $\mathrm{R} 2$ analog for the regression model was 0.7 or $70 \%$.

Use of biologics (coefficient $0.03,95 \%$ CI $-0.01-0.07$, $\mathrm{p}=0.20$ ) and $\mathrm{CD}$ did not show a moderating effect on overall mortality (Fig. 2). The increasing proportion of UC showed a positive trend towards mortality; however, this was not significant (coefficient $0.07,95 \% \mathrm{CI}-0.01$ to -0.14 , $\mathrm{p}=0.08$ ). Increasing mean age showed a significantly positive trend towards mortality (coefficient $0.13,95 \% \mathrm{CI}$ $0.02-0.23, \mathrm{p}=0.02)$. The $\mathrm{R} 2$ analog was 1 or $100 \%$.

\section{Discussion}

We found the overall prevalence of "severe" COVID-19mechanical ventilation, ICU admission, and mortality, for all IBD patients with COVID-19 to be $5.1 \%, 6.1 \%$, and $4.5 \%$, respectively. This appears to be fairly low given the impact IBD and its medications may have on the immune-system. In fact, the increasing proportion of IBD patients showed a significantly negative association with the need of mechanical ventilation. Perhaps these findings can be explained by the consistent levels of ACE2 receptors in IBD patients compared to the general population [3]. Moreover, further therapy with immunosuppressants/biologics may decrease the expression of these molecules, resulting in overall reassurance for IBD patients [3].

We also found that the use of biologics did not predict "severe" COVID-19-as shown upon multivariable analysis from our meta-regression model. This finding is important for several reasons. First, it advocates for the ongoing and continued IBD therapy (biologics) in patients during the COVID-19 pandemic, as has been suggested based upon expert opinion [4]. Second, continuing IBD therapy will minimize flares requiring in office or in hospital evaluations, thus contributing to keeping IBD patients safe amidst the pandemic. Additionally, this finding is in line with our knowledge on biological medications, which seem to increase the risk of opportunistic infections, but do not perpetuate serious infections [2]. Furthermore, the hypothesis of suppressing the "cytokine storm" associated with severe COVID-19 may contribute to their benefit in this subset of patients [5].

Despite our important findings, several limitations must be considered [6]. First, some studies also included highly suspicious cases in their COVID-19 cohort. Second, not all studies were of similar design, and thus, a direct comparison to the aforementioned outcomes was not the main focus of every study—likely leading to bias [6]. Fourth, it could not be determined if a study included some patients whom were already accounted for in another registry [6]. Finally, the concomitant use of thiopurine immunosuppressants or certain classes among the biologics may have different effects on COVID-19 outcomes-but was too sparsely reported to generate meaningful conclusive data.

In conclusion, the current evidence suggests that the IBD population is somewhat protected from developing severe COVID-19-as determined by the lack of adverse outcomes, compared to the general population. The protection is likely 
Fig. 2 Scatter plots demonstrating the impact of biologics on outcomes; A need for mechanical ventilation, B need for intensive care unit (ICU) admission, and $\mathbf{C}$ overall mortality
A Regression of Logit event rate on biologic

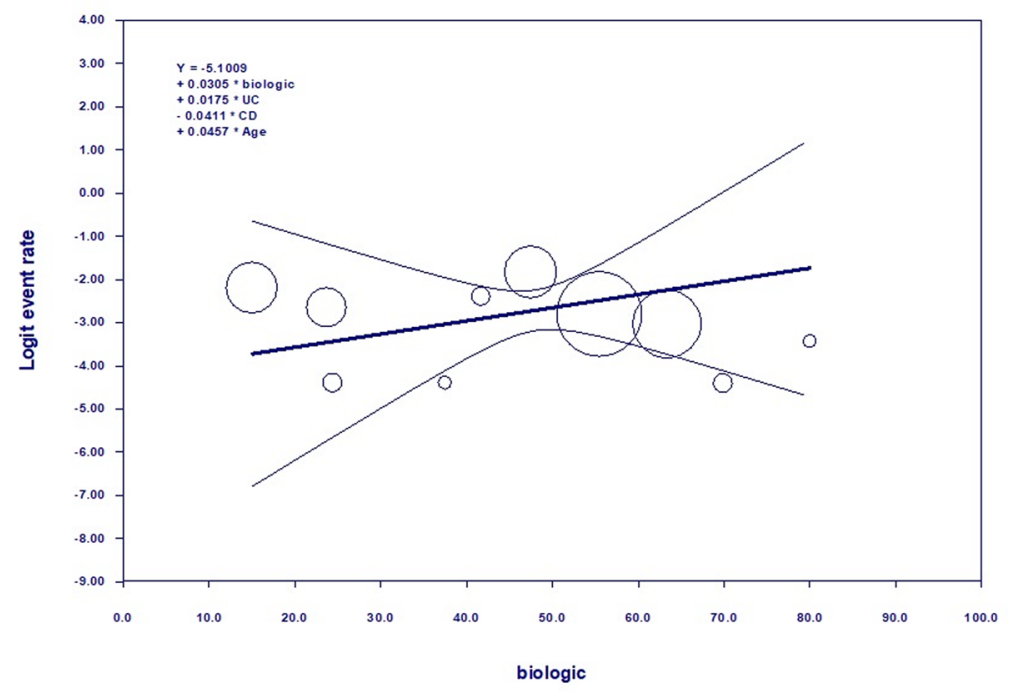

B

Regression of Logit event rate on biologic

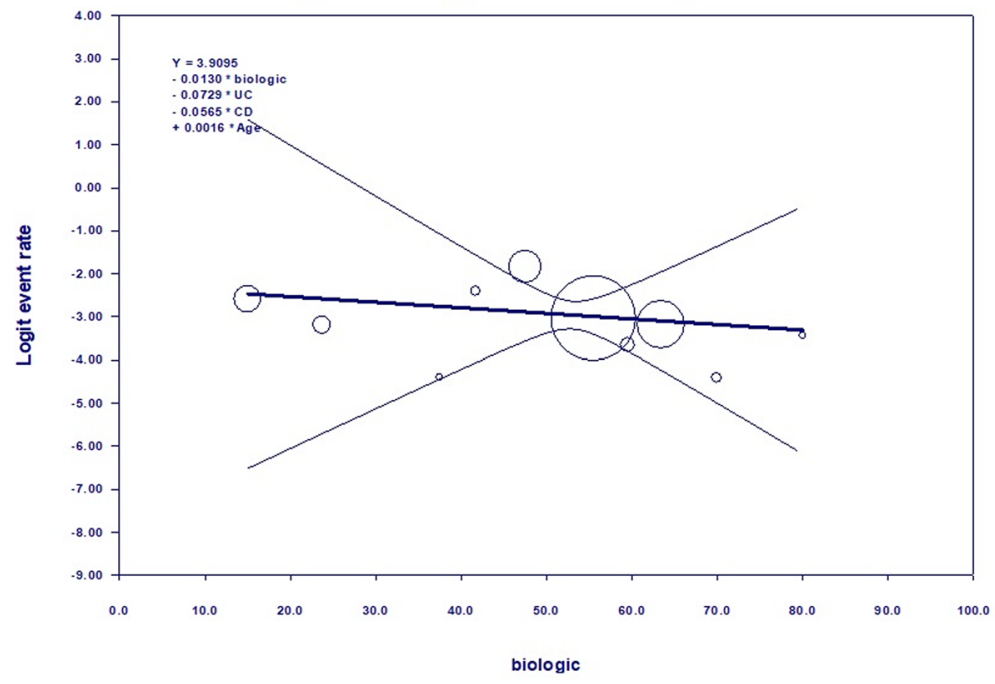

C

Regression of Logit event rate on biologic

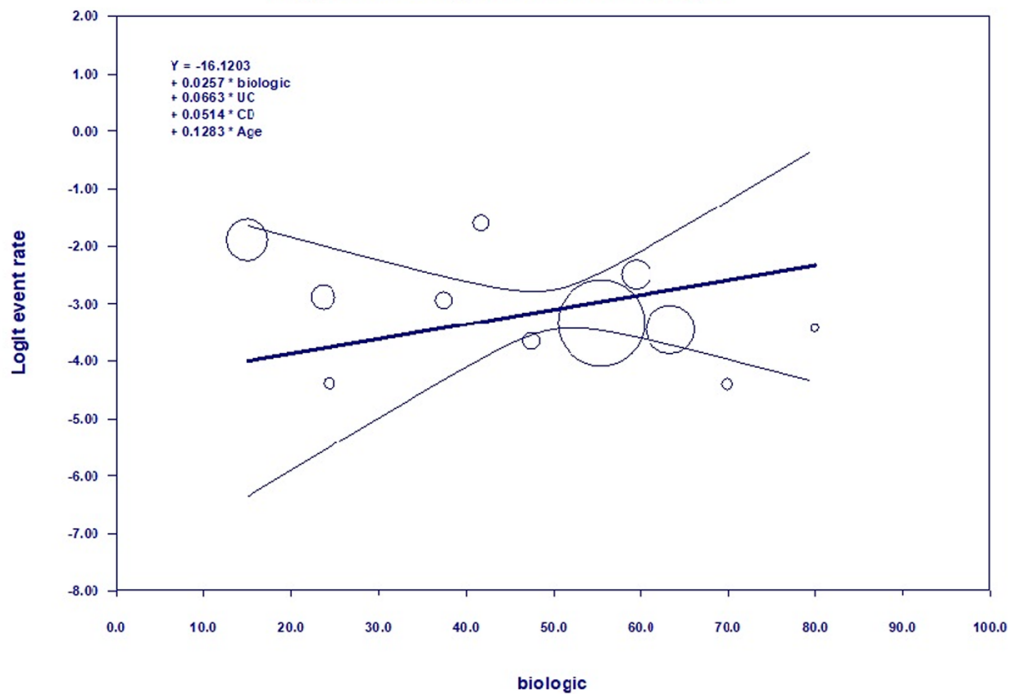


due to ongoing therapy of IBD and should not be discontinued. The incidence, severity, and outcomes related to COVID-19 in IBD patients' needs to be reassessed as data continues to emerge from the pandemic. Additional outcomes data will be required to understand how all classes of biologics and/or the use of concomitant immunosuppressant effect COVID-19 outcomes in IBD patients.

Author contribution SW, MA, and WLS assisted with data acquisition, analyses, and manuscript preparation. SW and MA critically revised the manuscript and provided input regarding methodology. SE, AS, and JDF provided direct supervision and guidance. SW is the article guarantor. All authors agree to the final version of this manuscript.

Data availability All data used herein is available either in the text and/or figures.

\section{Declarations}

Conflict of interest The authors declare no competing interests.

\section{References}

1. Zhou P, Yang XL, Wang XG et al (2020) A pneumonia outbreak associated with a new coronavirus of probable bat origin. Nature 579:270-273
2. Bonovas S, Fiorino G, Allocca $M$ et al (2016) Biologic therapies and risk of infection and malignancy in patients with inflammatory bowel disease: a systematic review and network meta-analysis. Clin Gastroenterol Hepatol 14:1385-1397

3. Burgueño JF, Reich A, Hazime H et al (2020) Expression of SARS-CoV-2 entry molecules ACE2 and TMPRSS2 in the gut of patients with IBD. Inflamm Bowel Dis 26:797-808

4. Rubin DT, Feuerstein JD, Wang AY et al (2020) AGA Clinical practice update on management of inflammatory bowel disease during the COVID-19 pandemic: expert commentary. Gastroenterology 159(1):350-357

5. Zhong J, Tang J, Ye C, Dong L (2020) The immunology of COVID-19: is immune modulation an option for treatment? Lancet Rheumatol 2(7):e428-e436

6. Sultan K, Mone A, Durbin L, Khuwaja S, Swaminath A (2020) Review of inflammatory bowel disease and COVID-19. World J Gastroenterol 26(37):5534-5542

Publisher's Note Springer Nature remains neutral with regard to jurisdictional claims in published maps and institutional affiliations. 